\title{
Preventing Traumatic Urinary Catheter Insertion Through a Computerized Ordering System: Quasi- experimental Study From a Tertiary Academic Center
}

\author{
Ted Lee ${ }^{1 *}$, Heiko Yang' ${ }^{1}$ David A. Hanauer ${ }^{2}$, Julian Wan' \\ ${ }^{1}$ Department of Urology, University of Michigan, Ann Arbor, USA \\ ${ }^{2}$ Department of Computational Medicine and Bioinformatics, University of Michigan, Ann Arbor, USA
}

*Corresponding Author: Ted Lee, M.D., Department of Urology, University of Michigan, 1500 E. Medical Center
Drive, Ann Arbor, MI 48109, USA. Tel: +1-2015437520, Fax: +1-7342321610, Email: Ited@med.umich.edu

Received September 17, 2017; Accepted December 2, 2017; Online Published January 28, 2018

\begin{abstract}
Background: latrogenic urethral injury is a well-known risk of urinary catheter insertion. Associated morbidities include stricture formation, catheter associated infection, risk for instrumentation, and extended length of stay. Yet, insufficient attention is paid to its prevention.

Objective: This study aimed to assess the effectiveness of a computerized ordering system in reducing iatrogenic urethral injuries in men at high risk for iatrogenic urethral injury from traumatic catheterization.

Methods: A pre-post study was performed to assess the impact of a computerized ordering system that recommended a $16 \mathrm{Fr}$ Coudé-tip catheter be used instead of the standard 16 Fr Foley catheter for men at high risk for difficult catheter insertion. The intervention was implemented in October 2012. The rates of traumatic and non-traumatic insertion consults were compared between the pre-intervention (10/1/2010-09/30/2011) and post-intervention (10/1/2012-9/30/2013) time periods.

Results: The results showed that $78.5 \%$ of patients with iatrogenic urethral injury possessed at least one identified risk factor for difficult catheterization. There was no significant difference in frequency of traumatic or non-traumatic insertion consults between the pre- and post-intervention cohorts.

Conclusion: The current study highlights the possibility of preventing traumatic insertions by targeting men at high risk for iatrogenic injury. Without a simultaneous plan for improving catheter placement awareness and training, any change in the ordering process is unlikely to succeed on its own.
\end{abstract}

Keywords: Urinary Catheterization, Iatrogenic Disease, Urethra, Male

\section{Background}

"Nearly one fourth of hospitalized patients have an indwelling urethral catheter placed at some point during admission". Morbidity involving urethral catheter insertion is not trivial. Urothelial tears and false passage creations are extremely painful for patients and among the most common causes of anterior urethral injuries. ${ }^{2}$ One third of anterior strictures are attributed to iatrogenic urethral trauma. ${ }^{3}$ Other sequelae include gross hematuria, urinary retention, and urinary tract infection. ${ }^{4}$

Iatrogenic urethral injuries are costly. One third of genitourinary traumas secondary to insertion problems require some type of intervention. ${ }^{5}$ These complications increase the length of hospital stay and the risk for infection. ${ }^{6}$ The complete extent of morbidities is likely underestimated, because complications, such as urethral strictures and meatal stenosis, manifest long after the inciting event.

Many iatrogenic urethral injuries are preventable. ${ }^{7}$ Yet, prevention strategies that target those with the highest risk for iatrogenic trauma are few. Patients who are at the greatest risk for difficult urethral catheter insertions are men with benign prostatic hyperplasia $(\mathrm{BPH})$ and a history of other known urologic issues. ${ }^{8}$ Urologists typically take a proactive approach in managing these patients by using the Coudé-tip or other catheters during insertion attempts. ${ }^{9}$ However, such maneuvers are not routinely performed by the non-urological personnel who place the vast majority of catheters.

A University of Michigan urology group implemented a computerized clinical decision support system aimed at reducing iatrogenic urethral injuries in high-risk men. A set of questions were developed from an earlier study that assessed the common risk factors in men who required

Copyright (C) 2018 The Author(s). This is an open-access article distributed under the terms of the Creative Commons Attribution License (http:// creativecommons.org/licenses/by/4.0), which permits unrestricted use, distribution, and reproduction in any medium, provided the original work is properly cited. 
difficult Foley catheterization consults. These questions were used to define men at higher than usual risk. ${ }^{10}$

\section{Objective}

On the basis of the answers to these questions, providers were prompted to use a Coudé-tip catheter for men identified as high-risk. Providers were also instructed to promptly seek a urology consult after two unsuccessful attempts to get ahead of significant urethral swelling that can make subsequent catheter insertion very difficult. In the current study, it was hypothesized that the intervention would lead to fewer episodes of traumatic catheter insertions at the cost of an increased number of consultations regarding difficult catheter insertions.

\section{Methods}

This pre-post study was performed to assess the impact of a computerized ordering system on the rates of iatrogenic urethral injuries.

\subsection{Intervention}

In the University of Michigan electronic medical record system (CareLink ${ }^{\mathrm{TM}}$ ), a series of questions were implemented to appear whenever a catheter placement order was initiated. The questions (listed below) screened for men at high risk of iatrogenic injury among those consulting for difficult catheterizations. ${ }^{10}$

A. Is the patient 60 years old or older?

B. Does the patient carry the diagnosis of $\mathrm{BPH}$ ?

C. Does the patient carry the diagnosis of prostate cancer?

D. Is the patient on home intermittent catheterization?

E. Is the patient taking BPH drugs (alpha blockers - e.g., prazosin; 5 alpha-reductase inhibitors - e.g., finasteride)? If the answer to any question was YES, a recommendation that a 16 French (Fr) Coudé-tip catheter be used instead of the standard 16 Fr Foley would appear. The provider was instructed to consult urology immediately after two unsuccessful attempts. This system was implemented on October 1, 2012, following Institutional Review Board Approval (Figure 1).

\subsection{Data Collection and Analysis}

Beginning in 2007, all consults seen by our urology department were prospectively recorded in a dedicated consult database. ${ }^{11}$ The consulting resident entered the information of each consult into the database. Each consultation entry that involved urinary catheter placement during the pre-intervention time period (10/1/2010$09 / 30 / 2011)$ and post-intervention time period (10/1/2012 - 9/30/2013) was reviewed.

The exclusion criteria were aged less than 18 years, female patients, catheter insertions occurring prior to arrival at our institution, self-inflicted urethral trauma, traumatic removal, and suprapubic catheter-related consults.

Based on a review of the database and through manual chart confirmation, traumatic insertions were differentiated from non-traumatic insertions. A traumatic

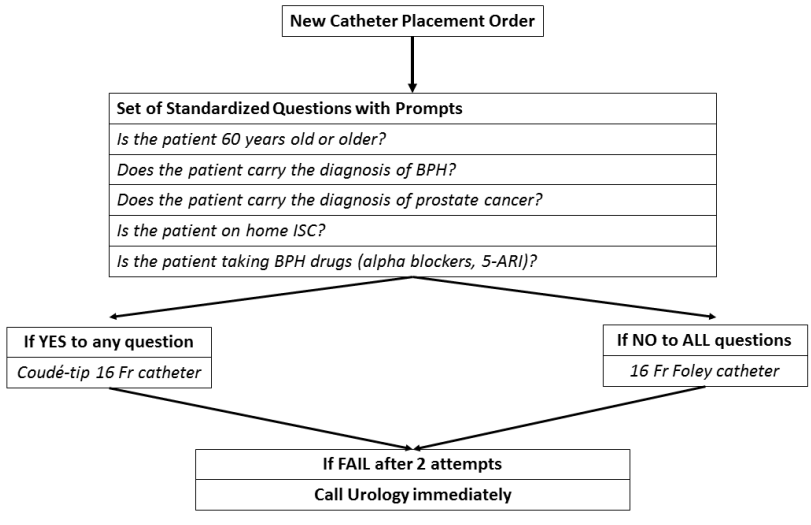

Figure 1. Computer Questions Flow Chart.

insertion injury consult was defined as occurring if the urology consultant documented at least one of the following conditions: significant blood at the urethral meatus, balloon inflation of a non-draining catheter that could not be irrigated, or cystoscopic evidence of urethral injury.

To examine the effects of the intervention, interventionrelated patient factors (patient age, existing diagnosis of $\mathrm{BPH}$, prostate cancer and history of therapy, recent history of non-traumatic catheterization), details of the consult, urologic assessment and decision-making, and outcome were quantified for all consults in the study groups. For statistical analysis, $P$ values were calculated using Fisher exact test, z-test, and $t$ test.

\section{Results}

There were 1482 total consults during the pre-intervention period (10/1/2010-9/30/2011) and 2035 consults during the post-intervention period (10/1/2012-9/30/2013). Among the 3517 urologic consults in total, 501 (14.2\%) involved urinary catheter issues. After the exclusion criteria were applied, there were 305 (8.8\%) consults for difficult urethral catheter insertions. Traumatic insertions occurred in $16.7 \%(51 / 305)$ of these consults; the rest (254/305) were considered non-traumatic catheter insertion consults.

The average age of all men requiring consultation for difficult catheter insertion was 63.3 years (SD 15.9 years). The percentages of men with $\mathrm{BPH}$, prostate cancer, and home intermittent catheterization were $23.6 \%$ (72/305), $16.0 \%$ (49/305), and $12.8 \%$ (38/305), respectively. Nearly $12 \%(36 / 305)$ of patients had a history of radical prostatectomy, and 9.5\% (29/305) of patients had a history of transurethral procedures for $\mathrm{BPH}$. One or more risk factors were found in $78.5 \%(239 / 305)$ of consults.

The consulting urology resident managed $70.6 \%$ $(36 / 51)$ of traumatic insertion consults by simple catheter insertion. A 20 Fr Coudé-tip catheter was the catheter most commonly used by the urology resident. Only 13.7\% (7/51) of trauma cases required bedside flexible cystoscopy. None of the patients required emergent cystoscopy or suprapubic placement in the operating room. More than one fourth 
Table 1. Comparison of Consults Before and After Intervention

\begin{tabular}{|c|c|c|c|}
\hline & Pre-intervention $(10 / 1 / 10-9 / 30 / 11)$ & Post-intervention (10/1/12 - 9/30/13) & $P$ Value \\
\hline Total number of consults & 1482 & 2035 & \\
\hline Consult for traumatic catheter placement & $1.5 \%(22 / 1482)$ & $1.4 \%(29 / 2035)$ & $0.88^{*}$ \\
\hline Consult for non-traumatic catheter placement & $6.6 \%(98 / 1482)$ & $7.7 \%(156 / 2035)$ & $0.23^{*}$ \\
\hline Age $>60$ & $63.6 \%(14 / 22)$ & $62.1 \%(18 / 29)$ & $>0.99$ \\
\hline Pre-existing BPH & $22.7 \%(5 / 22)$ & $17.2 \%(5 / 29)$ & 0.73 \\
\hline Prostate Cancer & $13.6 \%(3 / 22)$ & $0 \%(0 / 29)$ & 0.07 \\
\hline Home ISC & $9.1 \%(2 / 22)$ & $27.6 \%(8 / 29)$ & 0.16 \\
\hline BPH Drugs & $18.2 \%(4 / 22)$ & $13.8(4 / 29)$ & $>0.99$ \\
\hline "NO" to all questions & $22.7 \%(5 / 22)$ & $24.1 \%(7 / 29)$ & $>0.99$ \\
\hline Requiring bedside cystoscopy & $13.6 \%(3 / 22)$ & $13.8 \%(4 / 29)$ & $>0.99$ \\
\hline Length of Foley catheter & 7.5 days & 6.6 days & $0.53^{\circ}$ \\
\hline Mode of catheter size & 20 French & 20 French & \\
\hline Previous non-traumatic catheterization & $36.4 \%(8 / 22)$ & $69.0 \%(20 / 29)$ & 0.02 \\
\hline
\end{tabular}

of patients (14/51) who suffered from iatrogenic urethral injuries were discharged with an indwelling catheter and had their catheter in place for an average of 6.4 days (SD 4.2 days). Patients discharged with indwelling catheters had appointments for catheter removal and trial of void at discharge or home intermittent straight catheterization at a later date.

For non-traumatic insertion consults, 63.7\% (162/254) of patients were managed by urethral catheter insertion. A 20 Fr Coudé-tipped catheter was also the most commonly used catheter. Bedside flexible cystoscopy was required in $17.3 \%$ (44/254) of difficult catheter cases. Four patients required cystoscopy and three patients required suprapubic tube placements in the operating room. Twelve percent of patients were discharged with an indwelling catheter in place.

There was no significant difference in frequency of traumatic or non-traumatic insertion consults between the pre- and post-intervention cohorts $(P=0.88$ and $P=$ 0.23 , respectively). Among traumatic insertion consults, the post-intervention cohort contained a higher number of patients with previous non-traumatic catheterization either at home or during the current admission (69.0\% vs $36.4 \%, P=0.02$ ) (Table 1).

\section{Discussion}

Understanding past history and risk factors is a key step in iatrogenic urethral catheter injury prevention. Men with known or suspected BPH may be first approached with a larger Coudé-tip catheter as was attempted in the current study, but men with known urethral stricture due to previous prostatectomy may be best served with smaller catheters or flexible cystoscopy guided insertion as a first line approach. The current study highlights the possibility of preventing traumatic insertions by targeting men at high risk for iatrogenic injury. Nearly $80 \%$ of patients included in this study possessed at least one factor identified as a risk factor for difficult catheterization.

The findings of this study also reiterate the importance of the primary provider's skill and experience in avoiding preventable insertion injuries. Men who suffered from iatrogenic urethral injuries in the post-intervention cohort were more likely to have documented non-traumatic catheterizations, suggesting that patients and other providers were able to previously insert catheters without injury $(P=0.03)$. These findings may be attributable to the unfamiliarity of primary providers with proper Coudé catheter insertion techniques.

Ultimately, successful urethral catheter placement is largely dependent on the skill and experience of the person actually performing the procedure. Systematic educational programs involving in-service programs for nurses and interns have shown to effectively decrease the rate of iatrogenic trauma from insertion attempts as well as the rate of urinary tract infections. ${ }^{4,12,13} \mathrm{~A}$ training program to educate nurses, interns, and medical students on proper catheter insertion techniques including the use of Coudé catheters would have strengthened the intervention in this study. Efforts can also be made to ensure adherence to proper catheter insertion techniques and to minimize unnecessary urinary catheter insertion attempts. ${ }^{14,15}$

In addition, the provider entering the order often was not the one who actually performed the catheterization. Even if the questions were answered correctly and risk factors were flagged in the computerized ordering system, it is uncertain whether the nurse, intern, or medical student took the positive action of ordering a Coudé-tip catheter. Additional limitations of the current study include underreporting or under-diagnosis of iatrogenic urethral injuries. Only the traumatic catheterizations to which the authors were alerted are reported herein.

\section{Conclusion}

A set of simplified questions were designed and implemented in the computer ordering system in an attempt to prevent iatrogenic urethral injuries in high-risk patients. Although the frequency of iatrogenic urethral injuries remained unchanged after the intervention, the current study highlights the possibility of preventing traumatic insertions by targeting men at high risk for 


\section{Research Highlights}

\section{What Is Already Known?}

Urinary catheter insertion is a high-volume procedure that is routinely performed by healthcare providers. Morbidity involving a traumatic urinary catheter insertion is not trivial. Tissue tearing and false passage creation from traumatic catheter insertions are extremely painful for patients and among the most common causes of anterior urethral injuries. In addition, iatrogenic urethral injuries have been shown to increase the length of hospital stay and the risk for infection. Despite these risks, prevention strategies that target those with the highest risk for traumatic catheter insertions are few.

\section{What This Study Adds?}

A set of simplified questions were designed and implemented in the computer ordering system in an attempt to prevent iatrogenic urethral injuries in high-risk patients. Although the frequency of iatrogenic urethral injuries remained unchanged after the intervention, the current study highlights the possibility of preventing traumatic insertions by targeting men at high risk for iatrogenic injury. Without a simultaneous plan for improving catheter placement awareness and training, any change in the ordering process is unlikely to succeed on its own.

iatrogenic injury. Without a simultaneous plan for improving catheter placement awareness and training for the providers, house staff, nurses, medical students and aides of the primary service, any change in the ordering process is unlikely to succeed on its own.

\section{Authors' Contributions}

TL, HY, DH, and JW made substantial contributions to the concept and design; the acquisition, analysis, or interpretation of the data; drafting of the article or critical revision for important intellectual content; final approval of the version to be published; and agreement to be accountable for all aspects of the work, ensuring that questions related to the accuracy or integrity of any part of the article were appropriately investigated and resolved.

\section{Conflict of Interest Disclosures}

The authors declare that they have no conflicts of interest.

\section{Ethical Approval}

The researcher states ethical approval was gained from our institution and a protocol to maintain confidentiality and anonymity was applied (Ethical Committee Approval Number HUM00032231).

\section{References}

1. Haley RW, Hooton TM, Culver DH, et al. Nosocomial infections in U.S. hospitals, 1975-1976: estimated frequency by selected characteristics of patients. Am J Med. 1981;70(4):947-959. doi:10.1016/0002-9343(81)90561-1.

2. Dobrowolski ZF, Weglarz W, Jakubik P, Lipczynski W, Dobrowolska B. Treatment of posterior and anterior urethral trauma. BJU Int. 2002;89(7):752-754. doi:10.1046/j.1464410X.2002.02719.x.

3. Fenton AS, Morey AF, Aviles R, Garcia CR. Anterior urethral strictures: etiology and characteristics. Urology. 2005;65(6):1055-1058. doi:10.1016/j.urology.2004.12.018.

4. Kashefi C, Messer K, Barden R, Sexton C, Parsons JK. Incidence and prevention of iatrogenic urethral injuries. J Urol. 2008;179(6):2254-2257; discussion 2257-2258. doi:10.1016/j. juro.2008.01.108.

5. Leuck AM, Wright D, Ellingson L, Kraemer L, Kuskowski MA, Johnson JR. Complications of Foley catheters--is infection the greatest risk? J Urol. 2012;187(5):1662-1666. doi:10.1016/j. juro.2011.12.113.

6. Aaronson DS, Wu AK, Blaschko SD, McAninch JW, Garcia M. National incidence and impact of noninfectious urethral catheter related complications on the Surgical Care Improvement Project. J Urol. 2011;185(5):1756-1760. doi:10.1016/j.juro.2010.12.041.

7. Buddha S. Complication of urethral catheterisation. Lancet. 2005;365(9462):909. doi:10.1016/s0140-6736(05)71051-5.

8. Liu JJ, Guo DP, Gill H. Patterns of urinary catheter consults in a tertiary care hospital. Can J Urol. 2013;20(6):7046-7049.

9. Villanueva C, Hemstreet GP 3rd. The approach to the difficult urethral catheterization among urology residents in the United States. Int Braz J Urol. 2010;36(6):710-715. doi:10.1590/ S1677-55382010000600009.

10. Styn N, Latini JM, Crossley H, Wan J. Evaluating The Impact Of Male latrogenic Urethral Injuries During Urinary Catheterization. J Urol. 2012;187(4):e380. doi:10.1016/j. juro.2012.02.1031.

11. Johnson EK, Filson CP, Faerber GJ, Park JM, Bloom DA, Wan J. Prospective tracking of pediatric urology consults: knowing is half the battle. J Urol. 2012;187(5):1844-1849. doi:10.1016/j. juro.2011.12.097.

12. Sullivan JF, Forde JC, Thomas AZ, Creagh TA. Avoidable iatrogenic complications of male urethral catheterisation and inadequate intern training: a 4-year follow-up post implementation of an intern training programme. Surgeon. 2015;13(1):15-18. doi:10.1016/j.surge.2014.02.001

13. Thomas AZ, Giri SK, Meagher D, Creagh T. Avoidable iatrogenic complications of urethral catheterization and inadequate intern training in a tertiary-care teaching hospital. BJU Int. 2009;104(8):1109-1112. doi:10.1111/j.1464410x.2009.08494.x.

14. Taleschian-Tabrizi N, Farhadi F, Madani N, Mokhtarkhani M, Kolahdouzan K, Hajebrahimi S. Compliance With Guideline Statements for Urethral Catheterization in an Iranian Teaching Hospital. Int J Health Policy Manag. 2015;4(12):805-811. doi:10.15171/ijhpm.2015.128.

15. Davoodian $P$, Nematee $M$, Sheikhvatan $M$. Inappropriate use of urinary catheters and its common complications in different hospital wards. Saudi J Kidney Dis Transpl. 2012;23(1):63-67. 